\title{
The COST Action TD1105: Lessons Learned and Outcome
}

\author{
Michele Penza ${ }^{1}$ - on behalf of EuNetAir Consortium \\ ${ }^{1}$ ENEA - Italian National Agency for New Technologies, Energy and Sustainable Economic \\ Development, Laboratory of Functional Materials and Technologies for Sustainable Applications, \\ Brindisi Research Center, PO Box 51 Br-4, I-72100 Brindisi, Italy \\ michele.penza@enea.it
}

\begin{abstract}
This is a short overview of the COST Action TD1105 EuNetAir - European Network on New Sensing Technologies for Air-Pollution Control and Environmental Sustainability - funded in the framework European Cooperation in the field of Scientific and Technical Research (COST) during the period 2012-2016. The international networking has involved more than 120 teams from academia, research, agencies and industry from 31 COST Countries and 7 Non-COST Countries (November 2016).
\end{abstract}

Key words: Sensing technologies, air pollution modelling, environmental measurements, methods and protocols, air quality control, environmental sustainability

\section{Introduction}

The main objective of the Concerted Action EuNetAir is to develop new sensing technologies for Air Quality Control (AQC) at integrated and multidisciplinary scale by coordinated research on nanomaterials, sensorsystems, air-quality modelling and standardised methods for supporting environmental sustainability with a special focus on Small and Medium Enterprises.

The COST Action TD1105 [1,2] - European Network on New Sensing Technologies for AirPollution Control and Environmental Sustainability - EuNetAir (see Fig. 1) aims to establish a top-level Pan-European multidisciplinary R\&D platform based on a new sensing paradigm for AQC contributing to sustainable development, green-economy and social welfare, and finally to create collaborative research teams in the European Research Area (ERA).

This international Networking, coordinated by ENEA (Italy), includes over 120 big institutions/organizations and over 200 international experts from 31 COST Countries (EU-zone: Austria, Belgium, Bulgaria, Croatia, Czech Republic, Denmark, Estonia, Finland, France, Germany, Greece, Hungary, Iceland, Ireland, Israel, Italy, Latvia, Luxembourg, The Former Yugoslav Republic of Macedonia, Netherlands, Norway, Poland, Portugal, Romania, Serbia, Slovenia, Spain, Sweden, Switzerland, Turkey, United Kingdom) and 7 Non-COST Countries (extra-Europe: Australia, Canada, China, Morocco, Russia, Ukraine,
USA) to create a S\&T critical mass in the environmental issues.

The challenges addressed by the COST Action TD1105 are listed as follows as:

- Nanomaterials for AQC sensors

- Low-cost gas sensors

- Low-power sensor-systems

- Wireless technology (Environmental Sensors Network)

- Air quality modelling

- Environmental measurements

- Standards and protocols

EuNetAir includes more than 200 experts and is mainly based on 4 Working Groups (WGs) and 4 Special Interest Groups (SIGs), coordinated by a leader:

$\checkmark$ WG1: Sensor materials \& nanotechnology

$\checkmark$ WG2: Sensors, devices \& systems for AQC

$\checkmark$ WG3: Environmental measurements \& airpollution modelling

$\checkmark$ WG4: Protocols \& standardisation methods

$\checkmark$ SIG1: Network of spin-offs

$\checkmark$ SIG2: Smart sensors for urban air monitoring in cities

$\checkmark$ SIG3: Guidelines for best coupling airpollutant and transducer

$\checkmark$ SIG4: Expert comments for revision of the Air Quality Directive (2008/50/EC)

Only a multidisciplinary cooperation will ensure cleaner air in Europe and reduced negative effects on human health for future generations in smart cities, efficient management of green buildings at low $\mathrm{CO}_{2}$ emissions, and sustainable economic development. 

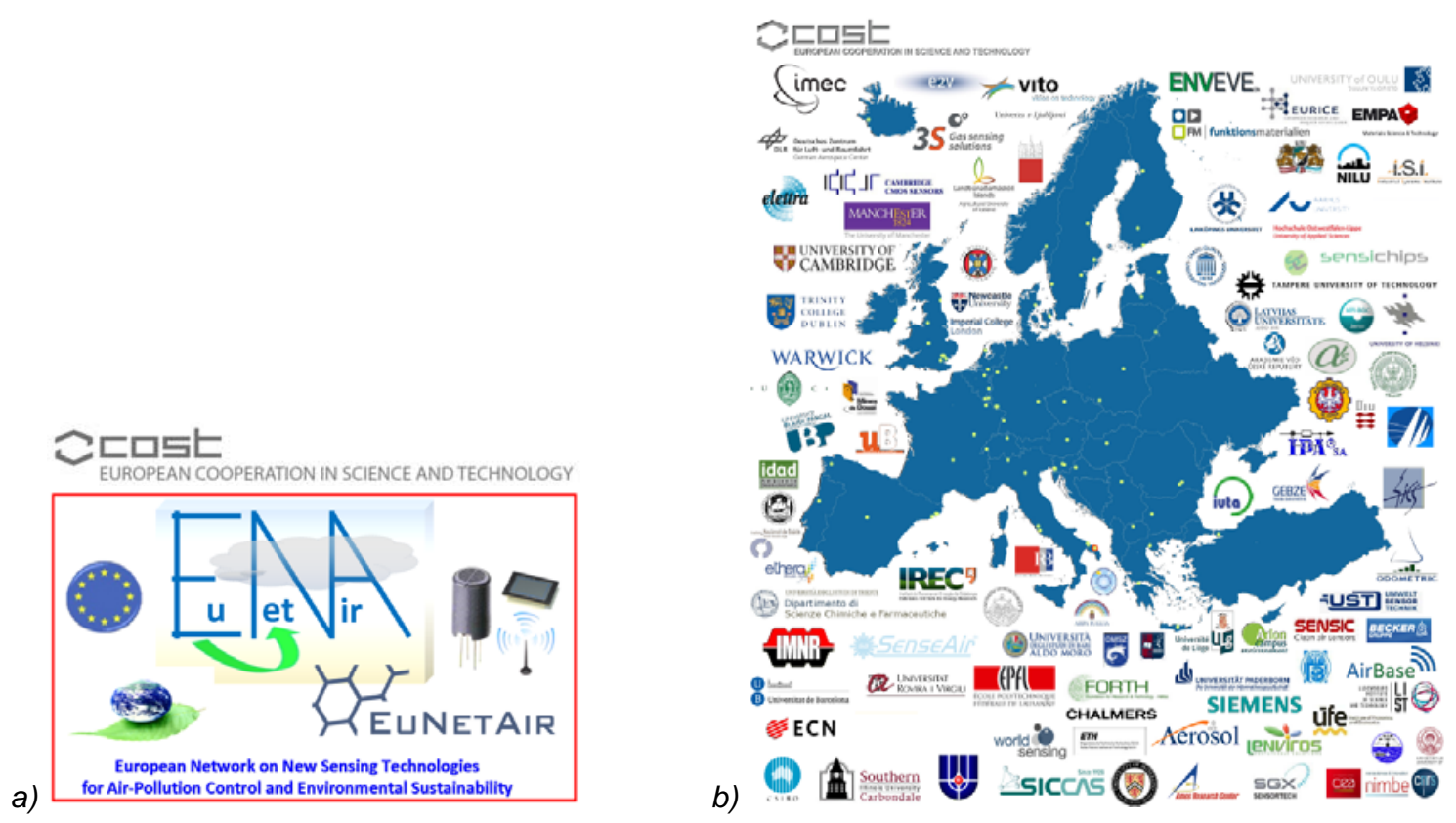

Fig. 1. The logo (a) and consortium (b) of COST Action TD1105. For further information: www.cost.eunetair.it.

\section{Short history of EuNetAir}

In the period 2012-2016, the COST Action EuNetAir [3-9] organized 28 Meetings (including 6 Scientific Meetings, 14 Focus/Core Group and Dissemination Meetings, 4 Working Group Meetings, 4 Workshops); 9 Management Committee Meetings, 4 Training Schools plus 2 Short Courses, and supported 43 European Early Career Investigators to carry out challenging research in the field of the environmental sensor technologies and measurements in foreign host institutions of the COST Countries and Associated Countries signing the Memorandum of Understanding (MoU) EuNetAir. Shortly:

\section{Scientific Meetings}

- Kick-off Meeting at COST Association, 16 May 2012, Brussels, Belgium

- $1^{\text {st }}$ Scientific Meeting at ENEA, 4-6 December 2012, Rome, Italy

- $2^{\text {nd }}$ Scientific Meeting at Queens' College, University of Cambridge, 18-20 December 2013, Cambridge, UK

- $3^{\text {rd }}$ Scientific Meeting at Bahcesehir University, GEBZE Technical University, 3-5 December 2014, Istanbul, Turkey

- $4^{\text {th }}$ Scientific Meeting at Linkoping University, 3-5 June 2015, Linkoping, Sweden

- $5^{\text {th }}$ Scientific Meeting at Bulgarian Academy of Sciences, 16-18 December 2015, Sofia, Bulgaria

- $6^{\text {th }}$ Scientific Meeting (Final Meeting) at Czech Academy of Sciences, 5-7 October 2016, Prague, Czech Republic
Workshops

- $1^{\text {st }}$ Workshop EuNetAir at IREC, joined to Transducers 2013 - Eurosensors 2013, 20 June 2013, Barcelona, Spain

- $2^{\text {nd }}$ Workshop EuNetAir at ENEA, 25-26 March 2014, Brindisi, Italy

- $3^{\text {rd }}$ Workshop EuNetAir at University of Latvia, 26-27 March 2015, Riga, Latvia

- $4^{\text {th }}$ Workshop EuNetAir at FFG, 25-26 February 2016, Vienna, Austria

WG Meetings

- $1^{\text {st }}$ WG Meeting EuNetAir at Fraunhofer Inhaus, 4-6 March 2013, Duisburg, Germany

- $2^{\text {nd }} W G$ Meeting EuNetAir at European Environment Agency, 3-5 October 2013, Copenhagen, Denmark

- $3^{\text {rd }}$ WG Meeting EuNetAir at IDAD, 13-15 October 2014, Aveiro, Portugal

- $4^{\text {th }}$ WG Meeting EuNetAir at VINCA Institute - University of Belgrade, 14-15 October 2015, Belgrade, Serbia

Training Schools

- $1^{\text {st }}$ Training School EuNetAir at University of Barcelona, Green Week 2013 Satellite Event, 13-15 June 2013, Barcelona, Spain

- $2^{\text {nd }}$ Training School EuNetAir at Saarland University, 31 March - 2 April 2014, Saarbrucken, Germany

- $3^{\text {rd }}$ Training School EuNetAir at University of Helsinki, 2-8 May 2015, Hyytiala (Helsinki), Finland 
- $4^{\text {th }}$ Training School EuNetAir at Aarhus University, 19-22 April 2016, Copenhagen, Denmark

- EuNetAir joined to Short Course ISOCS 2014 by Universitè Joseph Fourier, 9-14 February 2014, Les Houches, France

- EuNetAir joined to Short Course ISOCS 2015 by University of Brescia, 8-13 March 2015, Tonale (Brescia), Italy

Focus/Core Group and Dissemination Meetings

- EuNetAir at SGS-2012, 13 September 2012, Cracow, Poland

- EuNetAir at ISQL-2012, 29 September 2012, Halkidiki, Greece

- EuNetAir at TCM-2012, 21 October 2012, Hersonissos, Greece

- EuNetAir at EUROSENSORS-2014, 10 September 2014, Brescia, Italy

- EuNetAir at IEEE SENSORS 2014, 3 November 2014, Valencia, Spain

- Focus Group EuNetAir at Federal Environmental Agency - WHO Collaborating Centre, 17 April 2015, Berlin, Germany

- Focus Group EuNetAir at Siemens AG, 29 April 2015, Munich, Germany

- EuNetAir at ISOEN-2015, 28 June 2015, Dijon, France

- EuNetAir at EUROSENSORS-2015, 9 September 2015, Freiburg, Germany

- EuNetAir at AMA SENSOR+TEST-2016, 12 May 2016, Nuremberg, Germany

International Symposia and Conferences

- EuNetAir at European Materials Research Society (EMRS) Spring Meeting 2014, Symposium B: Advanced Functional Materials for Environmental Monitoring Applications, 26-30 May 2014, Lille, France

- EuNetAir at European Materials Research Society (EMRS) Spring Meeting 2016, Symposium X: Functional Materials for Environmental Sensors and Energy Systems, 2-6 May 2016, Lille, France

- EuNetAir at International Society of Exposure Science (ISES 2016), Advancing Exposure Science through Technology: Focus on Sensors, 9-13 October 2016, Utrecht, The Netherlands

- EuNetAir at Materials Research Society (MRS) Fall Meeting 2016, Symposium PM4: Novel Materials, Fabrication Routes and Devices for Environmental Monitoring, 27 November - 2 December 2016, Boston, USA

\section{Main results \\ International Networking}

This Action has successfully created a strong and qualified international networking devoted to the sensor technologies and environmental measurements/modelling including standards and protocols. In particular, the Action participants from COST Countries are 55 universities (44\%), 39 research centres (32\%), 4 environmental agencies (3\%) and 25 SMEs (21\%) including 9 spin-offs (8\%). Additional 8 top-level institutions from $\mathbf{7}$ Non-COST Countries such as 3 Near Neighbour Countries (NNC) - Morocco, Russia, Ukraine - and 4 International Partner Countries (IPC) Australia, Canada, China, USA - were involved in the Action as well.

This has created high impact with the establishing of an international network including about 15 SMEs and spin-offs. Several world-class experts from International Organizations such as European Environment Agency (EEA), World Health Organization (WHO) Europe, United Nations Economic Commission for Europe (UNECE) with group devoted to Long-Range Trans-Boundary Air Pollution - European Monitoring and Evaluation Programme (EMEP), Joint Research Center (JRC) - Institute for Environment and Sustainability, US Environmental Protection Agency (EPA), NASA Ames Research Center, MIT, CSIRO and Queensland University of Technology, have been involved with fruitful discussions and long-term cooperation.

\section{Significant Outcome}

This Action has produced significant results and deliverables as follows:

- Joint-exercise sensors-vs-analyzers in real city environment and related joint-publication

- Report on Innovation in sensor technology for air quality control

- Funded international (>2) and national (>10) research projects in air quality control

Low-cost gas and particulate sensors and lowpower consumption sensor-systems for air quality monitoring need repeated calibration. To improve accuracy and investigate long-term stability of their performance in real conditions, a joint-exercise was setup in Aveiro (Portugal), 13-27 October 2014 using an IDAD Air Quality Mobile Laboratory, equipped by reference analyzers, and parked in the city centre. The intercomparison was participated by 15 teams from 15 COST Countries make available 130 advanced low-cost sensors and sensorsystems. A joint-publication in a peer-review 
journal [10] at high impact factor has been coauthored by 29 EuNetAir members.

The Action will finalize a strategic report devoted to Innovation on environmental sensor technologies, expected on the 2016 end.

Furthermore, EuNetAir has been the networking platform to spin-out at least 5 international FP7 and H2020 projects such as SENSIndoor [11], MSP [12], IAQSense [13], CITI-SENSE [14] and iSCAPE [15] devoted to sensor-systems and their applications for air quality control. Moreover, EuNetAir affected the environmental research of the gas sensor-systems at national level in the Member States achieving funding for at least 10 national projects.

\section{Editorial Activities and Outreach}

The Action has produced 5 Special Issues to publish the most significant results, achieved by EuNetAir teams, in the Journal of Sensors and Sensor Systems (JSSS, Copernicus, 2014), Urban Climate (Elsevier, 2015), AMA Science Proceedings 2015, AMA Science Proceedings 2016, Beilstein Journal of Nanotechnology (Beilstein Institute, 2016).

The section VIDEO of the Action webpages [2] offers the interviews to 9 outstanding scientists and researchers from academia, research, agencies, international organizations and industry to outreach the EuNetAir topics for technical and non-technical target audience.

\section{Conclusions and Outlook}

EuNetAir is proposed to solve problems in the area of:

- Air Quality Control

- Environmental Sustainability

- Indoor/Outdoor Energy Efficiency

- Climate Change Monitoring

- Health Effects of Air-Pollution

EuNetAir is expected to impact as follows as:

- European Leadership on AQC Science \& Technology

- Development of Green-Economy

- Support to Sustainable Development

- Support to Monitoring System of Clean Air for Europe

- Fostering Research \& Innovation on New Sensing Technologies for Environmental Monitoring

Finally, some MC Members of the EuNetAir provided technical advising to the European Commission [16] in the field of sensor-systems at industrial relevance for $\mathrm{H} 2020$ inputs. This was compiled in the European Sensor Systems Cluster (ESSC) roadmap.

\section{Acknowledgements}

The EuNetAir consortium thanks to the COST Association for networking funding (COST Action TD1105) and the COST Officers for continuous support to manage this successful Action including Grant Holder Eurice (Germany) project managers. EuNetAir has been awarded by COST Association as a Top-Story [17].

\section{References}

[1] Action Memorandum of Understanding (MoU): www.cost.eu/domains_actions/essem/Actions/TD1105

[2] Action website: http://www.cost.eunetair.it

[3] M. Penza et al., COST Action TD1105: New Sensing Technologies for Environmental Sustainability in Smart Cities, IEEE Sensors 2014 Proceedings, Valencia, Spain, 2 - 5 November 2014. DOI: 978-1-4799-01623/14/\$31.00@2014 IEEE

[4] M. Penza, COST Action TD1105: Overview of SensorSystems for Air Quality Monitoring, Procedia Engineering 87 (2014) 1370-1377.

[5] M. Penza et al., COST Action TD1105: European Network on New Sensing Technologies for Air pollution Control and Environmental Sustainability, Linkoping EuNetAir Meeting, AMA Science 2015 Proceedings, Linkoping, Sweden, 3-5 June 2015. DOI 10.5162/4EuNetAir2015/01

[6] M. Penza, Portable Sensor-Systems for Air Quality Monitoring: The Case-Study of EuNetAir, $2^{\text {nd }}$ Meeting of the Global Platform on Air Quality and Health, Geneva, Switzerland, 18-20 August 2015.

[7] M. Penza, New Sensing Technologies for Environmental Sustainability Applications, The $5^{\text {th }}$ International WeBIOPATR Workshop and Conference Particulate Matter: Research \& Management, Belgrade, Serbia, 14-16 Oct. 2015

[8] M. Penza, COST Action TD1105: European Network on New Sensing Technologies for Air Pollution Control and Environmental Sustainability. Overview and Plans, Procedia Engineering 120 (2015) 476-479.

[9] M. Penza and T. Watkins, Advancing exposure science through technology: focus on sensors, International Society of Exposure Science, ISES 2016, Utrecht, the Netherlands, 9-13 October 2016.

[10] C. Borrego et al., Assessment of air quality microsensors versus reference methods: The EuNetAir joint exercise, Atmospheric Environment 147 (2016) 246-263.

[11] SENSIndoor - Nanotechnology-based intelligent multiSENsor System with selective pre-concentration for Indoor air quality control, www.sensindoor.eu

[12] MSP - Multi Sensor Platform for Smart Building Management, www.multisensorplatform.eu

[13] IAQSense - Nanotechnology-based sensors for environmental monitoring, www.iaqsense.eu

[14] CITI-SENSE - From sensor technology to citizen engagement, www.citi-sense.eu

[15] iSCAPE - Improving the Smart Control of Air Pollution in Europe, http://horizon2020projects.com/sc-climateaction/h2020-making-cities-sustainable

[16] Roadmap: Towards European Leadership in Sensor Systems; download at www.cluster-essc.eu

[17] EuNetAir awarded as Top-Story by COST Association; http://www.cost.eu/media/cost_stories/EuNetAir 